\title{
Critical Success Factors Affecting Implementation of Cloud ERP Systems: A Systematic Literature Review with Future Research Possibilities
}

\author{
Qian Huang* Mahbubur Rahim Susan Foster Misita Anwar \\ Monash University \\ Qian.Huang@monash.edu
}

\begin{abstract}
The implementation of Cloud Enterprise Resource Planning systems (Cloud ERP) is always challenging, which requires a variety of risks to be taken into consideration to ensure the success of the implementation. The assessment of Critical Success Factors (CSFs) in on-premise ERP implementations has been well documented but this research has not carried through to Cloud ERP implementations. Therefore, the contribution of this paper is to provide research and practice with identification and analysis of 35 CSFs through a systematic literature review. Drawing from the literature, we found security, project management, and communication are the top three widely cited CSFs during implementation. We also identify critical gaps in current research, such as inconclusiveness of findings related to CSFs and a lack of discussion on the nature of criticality of those CSFs. Furthermore, the attributes of CSFs are investigated in order to explore CSFs in a more logical and systematical way.
\end{abstract}

\section{Introduction}

In recent years, organizations worldwide have been adopting ERP systems operating in the cloud environment. Commonly known as Cloud ERP system, such systems are generally offered by drawing on the Software as a Service (SaaS) model. Thus, Cloud ERP systems are delivered over the Internet to multiple adopter organizations [1]. The proponents of Cloud ERP claim that adopter organizations can experience a range of benefits from operational benefits (e.g. reduced upfront costs, as well as cost savings, and faster implementation) through to strategic benefits (e.g. collaboration with supply chain partners, greater competitiveness [2]). According to a recent Cloud ERP market report, the market potential of cloud ERP is increasing and is expected to exceed USD 37.7 Billion by 2024 [3].

Given the growing acceptance and enormous market penetration potential of Cloud ERP, researchers from different disciplines are paying increased attention to various aspects of these systems. Drawing on $\mathrm{Yu}$ [4], the existing literature on Cloud ERP can be classified into three broad areas based on stages of innovation adoption: adoption, implementation, and post-implementation. Much of the existing literature focus on the organizational adoption of Cloud ERP [5]. Examples include the works of AlBar and Hoque [6] and Meghana, Mathew [7], to name a few. There also exists literature reviews (e.g. Salim [5]) reporting on the key themes associated with Cloud ERP adoption. In contrast, except for a few papers e.g., Peng and Nunes [8] Hasan, Miah [9], limited research has been reported to examine the post-implementation issues of Cloud ERP including critical processes such as testing and checking reliability of systems, or evaluating the benefits of system implementations.

Interestingly, research addressing: implementation issues (e.g. implementation model [10, 11], challenges $[12,13]$, and CSFs $[14,15])$ of Cloud ERP is gradually emerging. However, no systematic literature analysis of the existing studies on Cloud ERP implementation are reported. In this paper, our primary focus is on CSFs affecting the phenomenon of Cloud ERP implementation. Implementing a new system like Cloud ERP exerts many challenges and risks that need to be taken into consideration during the implementation process [16]. This is due to the success rate of the ERP implementation being very low, and a failure rate of up to $90 \%$ in some countries [17]. To successfully address the risks associated with Cloud ERP implementation, it is necessary to understand related CSFs [16].

While some researchers have endeavored to examine CSFs affecting cloud ERP implementation, their findings are fragmented, and to some extent inconclusive since these studies discussed CSFs from different perspectives and in different contexts. Hence, in this paper the scattered CSFs are rearranged in an organized and sensible set in order to achieve a fuller understanding of the uptake of Cloud ERP during implementation. 
Comprehensively considering CSFs affecting Cloud ERP implementation is highly significant to help organizations to successfully implement Cloud ERP and effectively reduce the occurrence of a wide variety of risks. Analyzing the existing literature will provide future research directions for scholars in the field. Therefore, our aim with this paper is twofold: first, to provide a platform for researchers and practitioners to recognize the complexity and absence of clarity about various CSFs affecting Cloud ERP implementation. Second, to identify the potential research issues associated with CSFs affecting Cloud ERP implementation that require further research investigation from the researcher community.

The paper is organized as follows: the methodology applied to conduct the systematic literature review is discussed in section two. The subsequent finding section is structured into four subsections based on various issues associated with CSFs affecting Cloud ERP. A discussion is presented in section four. Finally, conclusions as well as several directions for future research are offered.

\section{Methodology}

Our research, reported in this paper, was conducted by drawing on the principles of a systematic literature review [18] to identify CSFs of Cloud ERP implementation and generating detailed insights into them. Search, selection, analysis, and synthesis subprocesses involved in our review process are briefly described below.

\subsection{Search and selection}

The papers included in our review process met two criteria: a) only focus on Cloud ERP implementation, and b) they must have specifically used the term 'CSFs' in the title, abstract, keywords or body.

Our research began identifying papers by searching AIS "basket of eight" journals (e.g. Information Systems Journal, Information Systems Research, MIS Quarterly). To cover a broad a set of publications, and check the coverage, leading IS conferences (e.g. HICSS, ICIS, ECIS and AMCIS) and key journals of management and organization fields (e.g. OrgSc, OrgSt) were also searched.

Four steps were involved in the search and selection process. In step 1, journals and conferences were searched by using search terms: (Cloud ERP implementation) and (CSFs) or (factors) or (determinants). Several papers were excluded in Step 2 that did not meet the following criteria: first, the paper must be clearly related to our research (as mentioned in the Introduction section). Second, the paper must be written in English. Third, in order to obtain relatively recent content, the paper must have been published after 2010. Fourth, our research focuses only on conference papers and journal articles. Hence, other materials (e.g. Blogs, news items, workshop proposals) were excluded.

The main selection process focused on steps 3 and 4. In step 3 the initial selection was based on the title, abstract, and keywords. In step 4, the full texts of papers were manually assessed by four co-authors to ensure the chosen papers actually dealt with CSFs for the implementation of Cloud ERP. The following criteria were defined in order to select the candidate papers: first, CSFs for the success of Cloud ERP implementation are explicitly mentioned and are the focus of the papers' investigation. Second, Cloud ERP implementation does not refer to the organizational decision making of Cloud ERP adoption. Third, CSFs themselves are the primary focus or robustly mentioned. After Step 4, 10 papers were identified (shown in Table 1).

Table 1. The papers identified from the literature

\begin{tabular}{|c|c|c|}
\hline & Title & Year \\
\hline 1 & $\begin{array}{l}\text { SaaS enterprise resource planning } \\
\text { systems: challenges of their adoption } \\
\text { in SMEs[19] }\end{array}$ & 2013 \\
\hline 2 & $\begin{array}{l}\text { Critical success factors model for } \\
\text { business intelligent over ERP cloud } \\
\text { [20] }\end{array}$ & 2013 \\
\hline 3 & $\begin{array}{l}\text { Flexibility and improved resource } \\
\text { utilization through cloud based ERP } \\
\text { systems: critical success factors of } \\
\text { SaaS solutions in SME [21] }\end{array}$ & 2013 \\
\hline 4 & Implementation of Cloud ERP [22] & 2015 \\
\hline 5 & $\begin{array}{l}\text { Compliance, network, security and } \\
\text { the people related factors in cloud } \\
\text { ERP implementation [14] }\end{array}$ & 2016 \\
\hline 6 & $\begin{array}{l}\text { Moderating effect of compliance, } \\
\text { network, and security on the critical } \\
\text { success factors in the } \\
\text { implementation of cloud ERP [23] }\end{array}$ & 2016 \\
\hline 7 & $\begin{array}{l}\text { Perceived Use and Acceptance of } \\
\text { Cloud Enterprise Resource Planning } \\
\text { (ERP) Implementation in the } \\
\text { Manufacturing Industries[24] }\end{array}$ & 2016 \\
\hline 8 & $\begin{array}{l}\text { Role of cloud ERP and big data on } \\
\text { firm performance: a dynamic } \\
\text { capability view theory perspective } \\
\text { [25] }\end{array}$ & 2018 \\
\hline 9 & $\begin{array}{l}\text { Organizational, technological and } \\
\text { extrinsic factors in the } \\
\text { implementation of cloud ERP in } \\
\text { SMEs [15] }\end{array}$ & 2018 \\
\hline
\end{tabular}




\begin{tabular}{|l|l|l|}
\hline 10 & $\begin{array}{l}\text { Exploring Factors for Implementing } \\
\text { Cloud Enterprise Resource Planning } \\
\text { (ERP) Systems[26] }\end{array}$ & 2019 \\
\hline
\end{tabular}

As shown in Table 1, the majority of papers that met selection criteria were from IS journals and conferences, indicating to date most discussion resides within the IS community. The research about CSFs for the successful implementation of Cloud ERP is currently underrepresented since only ten papers have dealt with this research issue. Compared to literature review of CSFs in other related topic areas such as CSFs for ERP implementation (20 papers [27])), the success of Cloud ERP implementation literature has only marginally dealt with CSFs.

\subsection{Analysis and synthesis of the literature}

The analysis focused on summarizing and analyzing CSFs that have been discussed in Cloud ERP implementation, identifying supporting evidence and gaps in the literature [18]. As one of aims of this study is to provide new insights into the future research rather than only mapping the current discourse, a bottom up thematic analysis process was established. In line with the objectives, the following questions were used to guide analysis of the final ten papers: (1) what CSFs related to Cloud ERP implementation have been discussed (2) what are the complexity and absence of clarity about the various CSFs affecting Cloud ERP implementation. (3) potential research gaps associated with CSFs affecting Cloud ERP implementation that require further research.

\section{Findings}

A set of seven important topics associated with the CSFs affecting cloud ERP implementation are reported in the literature. Each topic is described in following seven sub-sections. Firstly, the frequency of the cited CSFs for the success of Cloud ERP implementation are presented. Secondly, CSFs are classified by category. Then, section three outlines and discusses the criticality and attributes of the CSFs. The methodology that has been used in the identified papers are discussed in section four. CSFs are discussed in terms of the organization size, the industry type, and countries in the last three sections respectively.

\subsection{Ranking of CSFs}

A total of thirty-five CSFs has been identified from literature analysis and are shown in Appendix-A. The most widely cited CSFs were found to be: security, project management, communication, compliance, network and the reaction of organization. These are described below.

The most widely cited CSF is security [14, 15, 19$21,23,28]$. Companies that move to cloud ERP have little knowledge about how cloud providers employ security platforms, processes and procedures, which may raise security risk or information leakage. The concerns, from the security point of view, when implementing Cloud ERP include: security breaches leading to confidential data being compromised, encryption, accountability, and maintenance issues [14]. Obviously, security breaches bring significant consequences to organizations; such as, leakage of user account details empowering attackers to access the confidential business information. As such, strategies, policies, security tools and mechanisms to ensure security play an important role in the successful adoption and implementation of Cloud ERP [15].

Project management is the second most widely cited CSF that is vital to successful implementation $[15,19,20,22,25,26]$. Project management involves multiple processes, which include initiating, planning, executing, and controlling. The variety of tools and techniques of project management methodologies are available to help organizations to overcome barriers faced by Cloud ERP implementation [26]. Furthermore, project teams, especially project leaders with the insightful knowledge of the domain can enhance the knowledge base of organizations, which also can assist organizations when they encounter challenges during the implementation [15].

Communication represents the third most widely cited CSF that is vital to successful implementation $[15,19,22,23,25]$. Effective communication can enrich employees' knowledge and can help employees learn from each other. Transparent and precise communication is required to reduce ambiguity in the understanding of employees during the implementation [23].

Compliance represents the next widely cited CSF $[14,15,20,23]$. Since organizations are uncertain about the means of data storage in the cloud, Cloudbased data archiving, segregation of duties, global compliance standards, and regulations are considered as the three major issues in compliance [23]. According to Gupta and Misra [14] and Gupta, Misra [15], high compliance will increase the possibility of successful implementation. Compliance also has a moderating effect between organizational factors and successful implementation of Cloud ERP [23].

Network is another frequently cited CSF that plays a crucial role in the implementation of cloud-based services as the high speed of the network is required to keep the systems updated with all the changes made by 
the users as well as via the cloud vendors $[14,15,20$, 23].

Moreover, the reaction of organizations to the change from on-premise to Cloud ERP is also critical for successful implementation. Organizations may face stiff challenge from their employees in migrating to Cloud ERP because they have little knowledge about benefits of Cloud ERP or are unwilling to changing the existing working habits or business processes [15]. Organization resistance often occurs when migrating from traditional ways of business operations to Cloud ERP solutions [23]. Therefore, organization resistance is considered as a widely cited CSF affecting successful implementation of Cloud ERP [15, 22, 23, $25]$.

\subsection{Types of CSFs}

In line with the taxonomy of CSFs proposed by Gupta and Misra [23], thirty-five CSFs are identified and presented in Table 2.

Table 2. Distribution of CSFs in terms of their types

\begin{tabular}{|c|c|c|c|}
\hline \multicolumn{3}{|c|}{ Intrinsic (32) } & Extrinsic(3) \\
\hline $\begin{array}{l}\text { Organizational CSFs } \\
\text { (15) }\end{array}$ & People CSFs (12) & $\begin{array}{c}\text { Technological CSFs } \\
\text { (5) }\end{array}$ & \\
\hline $\begin{array}{l}\text { - Communication } \\
\text { - } \text { - Organinization } \\
\text { resistance } \\
\text { - Project budget } \\
\text { - Project } \\
\text { management } \\
\text { - Implementation } \\
\text { - strategy } \\
\text { - Strategic goals and } \\
\text { objectives } \\
\text { - Business process re- } \\
\text { engineering } \\
\text { - organizational } \\
\text { culture } \\
\text { - knowledge base of } \\
\text { the company } \\
\text { - Project planning } \\
\text { - } \text { and control } \\
\text { Facilitating } \\
\text { conditions } \\
\text { - Organization size } \\
\text { The vision of the } \\
\text { - } \text { organization } \\
\text { Readiness of } \\
\text { - Crganizations } \\
\text { cority of potential } \\
\text { cost }\end{array}$ & $\begin{array}{l}\text { - User } \\
\text { involvement } \\
\text { - Selection of } \\
\text { vendor/consult } \\
\text { ant } \\
\text { - Project team } \\
\text { - Top } \\
\text { management } \\
\text { - support } \\
\text { - Training of user } \\
\text { - Trust on } \\
\text { vendor } \\
\text { - Performance } \\
\text { expectancy } \\
\text { - Effort } \\
\text { expectancy } \\
\text { - Social influence } \\
\text { - Age } \\
\text { - Gender } \\
\text { - Highest degree } \\
\text { attained }\end{array}$ & $\begin{array}{l}\text { - Selection of } \\
\text { ERP package } \\
\text { - IT } \\
\text { infrastructure } \\
\text { - Data integrity } \\
\text { and system } \\
\text { testing. } \\
\text { - Functionality } \\
\text { - System was } \\
\text { customized to } \\
\text { suite business } \\
\text { process }\end{array}$ & $\begin{array}{l}\text { - Security } \\
\text { - Compliance } \\
\text { - Network }\end{array}$ \\
\hline
\end{tabular}

This taxonomy of CSF is mainly for CSFs of Cloud ERP implementation and has been used in some Cloud ERP implementation literature. Out of thirty-five
CSFs, organizational and people types dominate, which contain 15 CSFs and 12 CSFs respectively. Five CSFs belong to the technological type. In summary, most of CSFs are related to intrinsic type whereas only three are related to extrinsic type, meaning most CSFs are under control of cloud ERP users.

Organizational CSFs are most widely mentioned. These CSFs (that are under the control of the adopter organization) can lead to the successful implementation of cloud ERP. For example, facilitating conditions refer to the extent of organizational support for the use of technology [24]. Next, people-oriented CSFs are also considerably mentioned. These factors are influenced by key stakeholders in the cloud ERP adopter organizations, such as, key users, and project team.

In contrast, technological CSFs and extrinsic CSFs are less frequently mentioned in the literature. Examples of CSFs which affect the implementation of cloud ERP from the technological point of view include IT infrastructure, and selection of ERP package.

Extrinsic factors include: compliance, network, and security as these CSFs are primarily controlled by the cloud vendor.

\subsection{Criticality and attributes of CSFs}

Existing Cloud ERP literature seriously lack a rich discussion on the nature of criticality of these CSFs. They mainly focus on investigating whether there are significant relationships among CSFs for successful implementation rather than discussing the strength of sufficiency as well as criticality (strong, weak) of those CSFs. Failure to solve the importance of CSFs adequately may lead to the failure or less than desired results [28].

In order to explore CSFs in a more logical and systematical way, and to understand interrelationships among CSFs and to address a successful implementation, Williams and Ramaprasad [29] developed a taxonomy of CSFs to distinguish a CSF from a non-CSF and their type and level of criticality. According to Williams and Ramaprasad [29], the taxonomy is based on four levels of criticality and three attributes of CSFs. Four levels of criticality include factors linked to success by a known causal mechanism; factors necessary and sufficient for success; factors necessary for success; and factors associated with success. The three attributes of CSFs are identified as standing or instigating, direct or indirect acting, and enhancing or inhibiting.

3.3.1 Types of criticality of CSF. Drawing on the notion of "types of criticality of CSF"[29], it is noted 
that no authors, who investigated CSFs for Cloud ERP implementation context, have explicitly discussed how critical those CSFs are. They mainly focus on investigating whether there are significant relationships among CSFs for a successful implementation. For example, Gupta and Misra [14] analyzed statistics and found network, compliance, security is positively related to the success of Cloud ERP implementation. In another study, Adeboye [24] only investigate whether performance expectancy, effort expectancy, social influence, facilitating conditions affect the success of Cloud ERP implementation. It is important to identify the strength of sufficiency as well as criticality (strong, weak) of those CSFs. This is because the CSFs that most strongly drive performance should receive priority attention by management as they facilitate the success of Cloud ERP implementation [30].

3.3.2. Attributes of CSFs. Attributes of CSFs are categorized by the role they play in ERP cloud implementation: the standing CSF refers to the part of the environment or background necessary for the success whereas the instigating CSF represents the trigger that make a difference or change in the background. A direct CSF represents it directly affect the success whereas an indirect CSF is itself related to success indirectly. Enhancing CSF refers to a CSF that enhances the possibility of the success. A CSF decreases the chance of the success can serve as the inhibiting CSF [29]. The aim of attributes of CSFs is to provide the understanding of the criteria related to the success of a project for executives by examining different aspects that affect each criterion in terms of time, connection and direction [31]. For example, through time property (standing or instigating), executives can understand whether some CSFs can directly trigger the success of an IT project or whether they serve as the background to support factors that affect success of an IT project [32].

Based on the notion of "the attributes of CSF" [29], it is noted that no researchers, who investigated CSFs for Cloud ERP implementation context, have explicitly discussed what the attributes of those CSFs are based on these three aspects. We have, after carefully analysing the findings from the final ten papers, described the characteristics of each CSF in terms of the three attributes as previous discussed. These are shown in Table 3.

\section{Table 3. Characteristics of CSFs}

\begin{tabular}{|c|c|c|c|c|}
\hline Factor type & Factor & \begin{tabular}{|l|} 
Standing/ \\
Instigating \\
\end{tabular} & Direct/Indirect & \begin{tabular}{|l|} 
Enhancing/ \\
Inhibiting \\
\end{tabular} \\
\hline \multirow[t]{12}{*}{ People } & \begin{tabular}{|l|} 
User involvement \\
\end{tabular} & Standing & Direct & Enhancing \\
\hline & Selection of vendor/consultant & Standing & Direct & Enhancing \\
\hline & \begin{tabular}{|l|} 
Project team \\
\end{tabular} & Standing & Direct & Enhancing \\
\hline & \begin{tabular}{|l|} 
Top management support \\
\end{tabular} & Standing & Direct & Enhancing \\
\hline & \begin{tabular}{|l|} 
Training of user \\
\end{tabular} & Standing & Direct & Enhancing \\
\hline & \begin{tabular}{|l|} 
Trust on vendor \\
\end{tabular} & Standing & Direct & Enhancing \\
\hline & \begin{tabular}{|l|} 
Performance expectancy \\
\end{tabular} & Not explicit & Not explicit & Not explicit \\
\hline & Effort expectancy & Not explicit & Not explicit & Not explicit \\
\hline & \begin{tabular}{|l|} 
Social influence \\
\end{tabular} & Not explicit & Not explicit & Not explicit \\
\hline & \begin{tabular}{|l|} 
Age \\
\end{tabular} & Not explicit & Not explicit & Not explicit \\
\hline & Gender & Not explicit & Not explicit & Not explicit \\
\hline & Highest degree attained & Not explicit & Not explicit & Not explicit \\
\hline \multirow[t]{15}{*}{ Organizational } & \begin{tabular}{|l}
$\begin{array}{l}\text { Communication within the } \\
\text { company }\end{array}$ \\
\end{tabular} & Standing & Direct/Indirect & Enhancing \\
\hline & \begin{tabular}{|l|} 
Organization resistance \\
\end{tabular} & Standing & Direct/Indirect & Inhibiting \\
\hline & \begin{tabular}{|l|} 
Project budget \\
\end{tabular} & Standing & Indirect & Enhancing \\
\hline & \begin{tabular}{|l|} 
Project management \\
\end{tabular} & Standing & Indirect & Enhancing \\
\hline & \begin{tabular}{|l|} 
Implementation strategy \\
\end{tabular} & Standing & Indirect & Enhancing \\
\hline & \begin{tabular}{|l|} 
Strategic goals and objectives \\
\end{tabular} & Standing & Indirect & Enhancing \\
\hline & Business process re-engineering & Standing & Indirect & Enhancing \\
\hline & \begin{tabular}{|l|} 
organizational culture \\
\end{tabular} & Standing & Direct & Enhancing \\
\hline & \begin{tabular}{|l|} 
knowledge base of the company \\
\end{tabular} & Standing & Direct & Enhancing \\
\hline & Organization size & Standing & Indirect & Enhancing \\
\hline & \begin{tabular}{|l|} 
The vision of the organization, \\
\end{tabular} & Standing & Indirect & Enhancing \\
\hline & \begin{tabular}{|l|} 
Project planning and control \\
\end{tabular} & Standing & Direct & Enhancing \\
\hline & \begin{tabular}{|l|} 
Readiness of organizations \\
\end{tabular} & Standing & Direct & Enhancing \\
\hline & Clarity of potential cost & Standing & Direct & Enhancing \\
\hline & \begin{tabular}{|l|} 
Facilitating conditions \\
\end{tabular} & Not explicit & Not explicit & Not explicit \\
\hline \multirow[t]{5}{*}{ Technological } & Selection of ERP package & \begin{tabular}{|l|} 
Standing \\
\end{tabular} & Indirect & Enhancing \\
\hline & IT infrastructure & Standing & Indirect & Enhancing \\
\hline & \begin{tabular}{|l|} 
Data integrity and system testing. \\
\end{tabular} & Standing & Indirect & Enhancing \\
\hline & \begin{tabular}{|l|} 
Functionality \\
\end{tabular} & Standing & Indirect & Enhancing \\
\hline & \begin{tabular}{|l|} 
System was customized to suite \\
business process \\
\end{tabular} & Standing & Indirect & Enhancing \\
\hline \multirow[t]{3}{*}{ Extrinsic } & Security & \begin{tabular}{|l|} 
Standing \\
\end{tabular} & Direct/Indirect & Enhancing \\
\hline & Compliance & Standing & \begin{tabular}{|l|} 
Direct// \\
Indirect
\end{tabular} & Enhancing \\
\hline & Network & Standing & Direct/Indirect & Enhancing \\
\hline
\end{tabular}

The majority of CSFs are categorized into standing factors. For example, according to Gupta and Misra [14], user involvement refers to employees of organizations who should be involved during the implementation stage to be familiar with Cloud ERP systems. The systems can be utilized in an optimum way to enhance the success of the Cloud ERP implementation only when users understand the benefits of these systems. Accordingly, involvement of employees can play an important role in producing an environment to enhance the success of Cloud ERP implementation, meeting the definition of a standing attribute. Top management support is also a standing CSF since it sets an environment in the organization to encourage and motivate employees to achieve strategic goals.

A further eleven CSFs are identified as direct factors since they are found to directly influence the success of Cloud ERP implementation [29]. In contrast, twelve CSFs are indirect factors as they affect success indirectly via other factors [29]. However, some CSFs are not only direct factors but also indirect factors. For example, Gupta and Misra [23] found the moderating effects of security, compliance and 
network on the relationship between organizational factors and successful implementation of Cloud ERP. The indirect influences of security, compliance and network on the success implementation are also identified from the study of Gupta, Misra [15].

Most CSFs are classified as enhancing factors, which means they increase probability of success [29]. For example, a competitive and skilled project team play an important role in successful implementation of Cloud ERP since the team can justify and achieve the organizational strategic and objectives [14]. While organization resistance is classified into inhibiting factors since it decreases the probability of a successful Cloud ERP implementation [29].

\subsection{CSFs and methodology}

Out of ten, seven papers conducted a survey (i.e. questionnaires) in their research design to investigate CSFs in Cloud ERP implementation [14, 15, 19, 23, 24, 26, 33]. For example, Gupta, Misra [15] surveyed different perspectives from cloud users and cloud vendors' in India. Whereas Adeboye [24] surveyed perspectives of cloud users and consultants. Lewandowski, Salako [19] collected data through formal interview and questionnaires from the users' perspective. The remaining three papers, Gupta and Misra [22], Emam [20], and Gerhardter and Ortner [21] all identified CSFs from the extant literature by reviewing existing theories (e.g. contingency theory, Social Capital Theory). For example, organization resistance was extracted from contingency theory since resistance would hinder transitioning to the usage of Cloud ERP.

\subsection{CSFs and organization size}

CSFs affecting cloud ERP implementation have been largely discussed in the context of SMEs. For instance, authors like Gupta and Misra [14], Gupta and Misra [23], Gupta, Misra [15], Gerhardter and Ortner [21], Gupta, Kumar [25], Emam [20], and Lewandowski, Salako [19] reported CSFs in the SME context as they play a significant role in promoting the economy of a country and thus constitute the core of economic growth. Some frequently cited CSFs are shown in Table 4.

Table 4. Common CSFs in SMEs

\begin{tabular}{|l|l|}
\hline CSFs & Sources \\
\hline Security & {$[14],[23],[15],[19]$,} \\
& {$[28],[20],[21]$} \\
\hline Project management. & {$[25],[15],[22],[19],[26]$,} \\
& {$[20]$} \\
\hline Communication within the & {$[23],[25],[15],[22],[19]$} \\
\hline
\end{tabular}

\begin{tabular}{|l|l|}
\hline company & \\
\hline Compliance & {$[14],[23],[15],[20]$} \\
\hline Network & {$[14],[23],[15],[20]$} \\
\hline Organization resistance & {$[23],[15],[22],[25]$} \\
\hline Project budget & {$[25],[15],[22],[19]$} \\
\hline Top management support & {$[14],[26],[25],[21]$} \\
\hline Training of user & {$[14],[21],[19],[26]$} \\
\hline $\begin{array}{l}\text { Data integrity/quality and } \\
\text { system testing. }\end{array}$ & {$[15],[21],[19],[26]$} \\
\hline Implementation strategy & {$[15],[26],[20],[21]$} \\
\hline $\begin{array}{l}\text { Strategic goals and } \\
\text { objectives }\end{array}$ & {$[15],[22],[26],[25]$} \\
\hline User involvement & {$[15],[25],[14],[19]$} \\
\hline $\begin{array}{l}\text { Business process re- } \\
\text { engineering }\end{array}$ & {$[15],[25],[22],[21]$} \\
\hline $\begin{array}{l}\text { Selection of } \\
\text { vendor/consultant }\end{array}$ & {$[14],[19],[26],[25]$} \\
\hline IT infrastructure. & {$[15],[25],[19]$} \\
\hline Functionality & {$[15],[25],[20]$} \\
\hline Project team & {$[14],[26],[25]$} \\
\hline Trust on vendor & {$[14],[26],[25]$} \\
\hline Selection of ERP package & {$[25],[15]$} \\
\hline Performance expectancy & {$[24],[20]$} \\
\hline Clarity of potential cost & {$[26],[20]$} \\
\hline & \\
\hline
\end{tabular}

For example, Gupta and Misra [14], Gupta and Misra [23], Emam [20] and Gupta, Misra [15] found compliance contributes to the successful implementation for SMEs.

However, several other authors like Lewandowski, Salako [19], Alharthi, Shehab [26] in their investigation of CSF did not explicitly mention organization size in their research. To date, no studies have reported CSFs in the context of large organizations. Hence, the applicability of those CSFs found relevant for the SME context cannot be generalized across to large organizations without further investigation. The size of the organization is significant to ERP implementation since expectations and requirements differ among SMEs and large organizations [16].

\subsection{CSFs and industry type}

The CSFs emerging from Cloud ERP implementation are reported to differ among organizations operating in different industries. Out of those ten papers in which CSFs were discussed, only two have described CSFs for a single industry. Adeboye [24] investigated successful implementation of cloud ERP in the manufacturing industry and reported no correlation between the performance expectancy, effort expectancy, social influence, facilitating conditions, with Cloud ERP implementation. Alharthi, Shehab [26] identified top management support, alignment of IT with business, 
and readiness of organizations as CSFs for technology organizations to implement Cloud ERP. Four papers, Gupta and Misra [14], Gupta, Misra [15], Lewandowski, Salako [19], Gupta, Kumar [25] discussed CSFs for multiple industries, such as banking, education, food industries. According to them, several CSFs (e.g. security, communication, project budget) are common across those industries. The authors of the remaining four papers did not mention industry type in their research.

\subsection{CSFs and country}

Studies investigating CSFs have been conducted in two specific countries: India and UK. For these two contexts, all CSFs were different. This was due to different theories being used by the authors to explain cloud ERP implementation in the two countries. For example, several theories were used to assess CSFs in Indian organizations: resource dependence theory (RDT), strategic choice theory, social capital theory, the contingency theory. In contrast, the unified theory of acceptance and use of technology (UTAUT) was applied to CSFs in UK. Alharthi, Shehab [26] investigated CSFs based on technology organizations from developing and developed countries.

\section{Discussion}

From the systematic literature review conducted into Cloud ERP implementation, we found that the top five most widely cited CSFs for the successful implementation of Cloud ERP are: security, project management, communication, compliance, and network. Lesser, but still important CSFs are organization resistance, project budget, implementation strategy, strategic goals and objectives, top management support, and training of users. Comparing these results with top $10 \mathrm{CSF}$ for on-premise ERP implementation identified by Leyh [34], factors differ but there exists some commonality. CSFs, such as communication, project management, strategic goals and objectives and top management support are important for both forms of ERP (i.e., on-premise, cloud-based). However, due to the nature of Cloud ERP, different CSFs are identified, leading to a successful implementation. For example, a high level of security and confidentiality is considered as one of the advantages of on-premise ERP, thus organizations usually do not consider security in their CSFs [35].

On the contrary, in Cloud ERP, sensitive information about organizations, financial data, such as bank details, are required to be shared with a thirdparty service provider(s) where data can be mingled with that of other companies. Because of the openness and multi-tenant feature of the cloud, organizations are concerned about how secure is their data and who has access [14, 15, 19-21, 23, 28]. Accordingly, security is considered as the most important CSF for the successful implementation from an organizations' perspective. This theme is in line with Hentschel, Leyh [16] who argued that security is the most frequently mentioned CSF by client organizations during the implementation and usage of cloud solutions. However, security serves as an extrinsic factor and can be controlled by vendors. According to Goel, Kiran [36], cloud providers have several resources to improve security solutions. For example, security of Cloud ERP systems can be guaranteed by applying standard encryption and decryption techniques. Furthermore, compared to on-premise ERP, Cloud ERP systems depend heavily on the high-speed quality of network: first, any failure of network and internet may disrupt the usual business function of client organizations [37]. Second, the high speed of the network is required to keep the systems updated with all the changes made by the users as well as the cloud vendors $[14,15,23]$. Accordingly, the network plays a crucial role in the implementation of cloud ERP.

Although some CSFs are widely cited in the extant literatures, the inconclusiveness of findings on CSFs exists. Three reasons explain this situation. Firstly, different positions of a variable (i.e. factors) contribute to the inconsistent findings. For example, the moderating effect of security on the relationship of technological factors and successful implementation of Cloud ERP has not been identified [23], whereas the impact of security on the implementation of Cloud ERP has been reported [15]. Secondly, different methodologies lead to differences in the significance of CSFs. For example, the influence of security on Cloud ERP implementation has been identified through a literature analysis and open-ended interview questions (e.g. Emam [20], Gerhardter and Ortner [21]), but the influence of security on Cloud ERP implementation does not exist through conducting a survey by using four indicators (i.e. confidentiality of data, encryption, accountability, maintenance) (e.g. Gupta and Misra [14]). Thirdly, the inconsistent findings about the same CSF results from different perspectives. For example, security is considered to have a positive relationship with Cloud ERP implementation in terms of multiple industries' perspective. On the contrary, it is not significant to Cloud ERP implementation in terms from a technology industry perspective (e.g. [15], [26]).

In terms of CSF taxonomy [24], relatively greater attention has been paid to organizational and people oriented CSFs. In contrast, technological and extrinsic factors are paid less attention in the existing research about CSFs in Cloud ERP implementations. This is 
perhaps because factors associated with the success or failure of ERP implementation related more to human and organizational factors rather than technical factors [38]. However, the reason why technological and extrinsic factors are least frequently cited is unknown, further study is thus needed to explore the reason and investigate whether they have a strong influence on Cloud ERP implementation as opposed to organizational and people-oriented CSFs through applying qualitative studies (e.g. in-depth case study).

While relationships among CSFs and successful implementation have been identified, the criticality of those CSFs has not been discussed. Failure to adequately solve CSFs based on their importance may lead to the failure or less than desired results (i.e. an unsuccessful implementation) [29]. As a result, further research should pay attention to investigate the importance of CSFs (i.e. Criticality of CSFs). According to the empirical results produced by Williams and Ramaprasad [29], attributes of CSFs show clear preferences: (a) instigating factors are preferred compared to standing factors; (b) direct factors are preferred compared to indirect factors; and (c) enhancing factors are preferred compared to inhibiting factors. The results of this research identify more enhancing factors than inhibiting factors, which is in line with the conclusion proposed by Williams and Ramaprasad [29].

Results of this research also indicate standing factors appear to be preferred than instigating factors and indirect factors appear to be preferred than direct factors, which conflict with the conclusion proposed by Williams and Ramaprasad [29]. This conflict can be explained by arguments of Williams and Ramaprasad [29]: first, biases of researchers and managers can result in this conflict. The research perspective and area that researchers focus on is different. Williams and Ramaprasad [29] discuss CSFs that are identified from the implementation of management information systems perspective whereas CSFs in this research are identified from Cloud ERP implementation perspective. Second, biased factors can be elicited by a biased framework whereas balanced factors can be elicited from a balanced framework since the managers and researchers would not seek some types of CSFs if those types were not part of their mental framework.

Several theories have been applied in the extant literature. Three theories: strategic choice theory, social capital theory, contingency theory, have received more attention for several reasons. For example, strategic choice theory is used by scholars to highlight the involvement of users during the implementation is critical, which is consistent with the emphasis of the theory. Social capital theory is used for the Cloud ERP context because it suggests that the support and commitment from the responsible leadership is crucial to set the environment in the organization. Contingency theory is employed as it emphasizes employees should be provided adequate training so that organizational resistance can be avoided during Cloud ERP implementation.

While, most of the existing papers only focus on the SMEs' context [14, 19-21, 23, 25], the size of the organization is a significant factor in assessing ERP implementation since expectations and requirements largely differ between SMEs and large organizations [39]. For example, migrating from an on-premise ERP to Cloud ERP can be a challenge and a heavy cost to large organizations as they operate large and complex infrastructure. Migration for SMEs is much easier since they have less complex structure, and are more flexible to adapt to the change $[39,40]$. However, few prior research studies have addressed the implementation of cloud-based ERP solutions in largesize organizations [13]. Accordingly, future research should pay attention to how CSFs contribute to the successful implementation of Cloud ERP in a large organizational context.

Clarification of some CSFs in relation to the organization size was conducted. For example, although security is considered the most important CSF, accordingly to Mac-Anigboro and Usoro [41] and Kiadehi and Mohammadi [42], large organizations would place more emphasis on the security of data and information compared to SMEs. However, a study conducted by Gupta, Misra [39] shows both large and small/medium sized organizations do not want to share important data with others, which means security and privacy of information is the top-most concern among both organizations. Therefore, further research should be conducted to establish whether security and privacy issues are a concern by all types of organizations or only large-scale organizations. Furthermore, existing research has focused on a limited number of countries, such as India [14, 15], and UK [19]. The results have some limitations as the data is only collected from one country the result might not hold true for other countries and regions [15]. Accordingly, we propose that further research should be conducted across other countries and regions.

\section{Conclusion and limitation}

The objective of this paper was to recognize the complexity and absence of clarity around various CSFs affecting Cloud ERP implementation. Of the available literature only ten papers were identified. These papers focused on CSFs that contribute to the success of Cloud ERP implementation. Thirty-five CSFs were 
identified through a systematic literature review and some interesting insights were provided. Firstly, the top six CSFs were identified: security, project management, communication, compliance, network, and organization resistance. Secondly, most of the existing papers only focus on an SMEs' context and only pay attention to limited countries such as India and the UK. Thirdly, most CSFs refer to organizational or people related factors rather than technological and extrinsic factors. Lastly, no scholars discuss the criticality and attributes of those CSFs in Cloud ERP implementation. The most important contribution for this research presents attributes of each CSF using the taxonomy of attributes identified by Williams and Ramaprasad [29]. This will aid researchers and practitioners to further establish CSFs to understand interrelationships among CSFs and logically and systematically. identify CSFs that support a successful implementation CSFs.

\section{References}

[1] Abd Elmonem, M.A., E.S. Nasr, and M.H. Geith, Benefits and challenges of cloud ERP systems-a systematic literature review. Future Computing and Informatics Journal, 2016. 1(1-2): p. 1-9.

[2] Vadivelu, K., et al., CLOUD-ERP: IMPLEMENTATION STRATEGIES, BENEFITS AND CHALLENGES. International Journal of Pure and Applied Mathematics, 2018. 119(14): p. 1359-1364.

[3] MarketsandMarkets(TM), Cloud ERP Market Worth $\$ 37.7$ Billion by 2024 - Exclusive Report by MarketsandMarkets(TM). 2019.

[4] Yu, C.S., Causes influencing the effectiveness of the post - implementation ERP system. Industrial Management \& Data Systems, 2005.

[5] Salim, S.A. Cloud ERP Adoption-A Process View Approach. in PACIS. 2013.

[6] AlBar, A.M. and M.R. Hoque, Factors affecting cloud ERP adoption in Saudi Arabia: An empirical study. Information Development, 2019. 35(1): p. 150-164.

[7] Meghana, H., A.O. Mathew, and L.L. Rodrigues, Prioritizing the factors affecting cloud ERP adoption-an analytic hierarchy process approach. International Journal of Emerging Markets, 2018.

[8] Peng, G.C. and M. Nunes, Establishing an evidencebased 9D evaluation approach for ERP post-implementation. Industrial Management \& Data Systems, 2017.

[9] Hasan, N., et al., Factors affecting postimplementation success of enterprise resource planning systems: a perspective of business process performance. Enterprise Information Systems, 2019. 13(9): p. 1217-1244.

[10] Kachur, R. and W. Kleinsmith, The evolution to the cloud-are process theory approaches for ERP implementation lifecycles still valid? Business Systems Review, 2013. 2(3): p. 72-93.

[11] Carutasu, N. and G. Carutasu, Cloud ERP implementation. FAIMA Business \& Management Journal, 2016. 4(1): p. 31.

[12] Rabaya, A. and K. Graffi. Implementing Enterprise Resource Planning Systems in the Cloud: Challenges and
Enabling CSFs that most strongly drive performance should receive priority attention by management and thus facilitating the success of Cloud ERP implementation. Our study also analyzed and identified a variety of research gaps in Cloud ERP implementation literature, thus providing a sound base for researchers and practitioners. For example, this research suggests future research should pay attention to the large organization context as the size of the organization is significant to ERP implementation. Further research should investigate the importance of CSFs (i.e. criticality of CSFs) to distinguish a CSF from a non-CSF. The main limitation of this study is that although the gap about criticality of CSFs is identified, it cannot be fully solved due to lack of the empirical study, thus the next step in our research is to conduct a quantitative approach to identify the criticality of CSFs.

Solutions. in 2019 International Symposium on Networks, Computers and Communications (ISNCC). 2019. IEEE.

[13] Sørheller, V.U., et al., Implementing cloud ERP solutions: a review of sociotechnical concerns. Procedia computer science, 2018. 138: p. 470-477.

[14] Gupta, S. and S.C. Misra, Compliance, network, security and the people related factors in cloud ERP implementation. International Journal of Communication Systems, 2016. 29(8): p. 1395-1419.

[15] Gupta, S., et al., Organizational, technological and extrinsic factors in the implementation of cloud ERP in SMEs. Journal of Organizational Change Management, 2018. 31(1): p. $83-102$.

[16] Hentschel, R., C. Leyh, and T. Baumhauer. Critical success factors for the implementation and adoption of cloud services in SMEs. in Proceedings of the 52nd Hawaii International Conference on System Sciences. 2019.

[17] Weng, F. and M.-C. Hung, Competition and challenge on adopting cloud ERP. International Journal of Innovation, Management and Technology, 2014. 5(4): p. 309.

[18] Jones, O. and C. Gatrell, The future of writing and reviewing for IJMR. International Journal of Management Reviews, 2014. 16(3): p. 249-264.

[19] Lewandowski, J., A.O. Salako, and A. Garcia-Perez. SaaS enterprise resource planning systems: challenges of their adoption in SMEs. in 2013 IEEE 10th International Conference on e-Business Engineering. 2013. IEEE.

[20] Emam, A.Z. Critical success factors model for business intelligent over ERP cloud. in 2013 International Conference on IT Convergence and Security (ICITCS). 2013. IEEE.

[21] Gerhardter, A. and W. Ortner, Flexibility and improved resource utilization through cloud based ERP systems: critical success factors of SaaS solutions in SME, in Innovation and Future of Enterprise Information Systems. 2013, Springer. p. 171-182.

[22] Gupta, S. and S.C. Misra, Implementation of Cloud ERP. 2015.

[23] Gupta, S. and S.C. Misra, Moderating effect of compliance, network, and security on the critical success factors in the implementation of cloud ERP. IEEE Transactions on Cloud Computing, 2016. 4(4): p. 440-451. 
[24] Adeboye, A., Perceived Use and Acceptance of Cloud Enterprise Resource Planning (ERP) Implementation in the Manufacturing Industries. International Journal of Strategic Information Technology and Applications (IJSITA), 2016. 7(3): p. 24-40.

[25] Gupta, S., et al., Role of cloud ERP on the performance of an organization. The International Journal of Logistics Management, 2018.

[26] Alharthi, S., E. Shehab, and A. Al-Ashaab. Exploring Factors for Implementing Cloud Enterprise Resource Planning (ERP) Systems. in Advances in Manufacturing Technology XXXIII: Proceedings of the 17th International Conference on Manufacturing Research, incorporating the 34th National Conference on Manufacturing Research, 10-12 September 2019, Queen's University, Belfast. 2019. IOS Press.

[27] Tarhini, A., et al., Analysis of the critical success factors for enterprise resource planning implementation from stakeholders' perspective: A systematic review. International Business Research, 2015. 8(4): p. 25-40.

[28] Alsharari, N.M., M. Al-Shboul, and S. Alteneiji, Implementation of cloud ERP in the SME: evidence from UAE. Journal of Small Business and Enterprise Development, 2020.

[29] Williams, J. and A. Ramaprasad, A taxonomy of critical success factors. European Journal of Information Systems, 1996. 5(4): p. 250-260.

[30] Finney, S. and M. Corbett, ERP implementation: a compilation and analysis of critical success factors. Business process management journal, 2007.

[31] Fitz-Gerald, L. and J. Carroll. The role of governance in ERP system implementation. in Proceedings of the 14th Australasian conference on information systems. 2003.

[32] Berman, U., Building a CSF framework for CRM implementation. Journal of Database Marketing \& Customer Strategy Management, 2009. 16(4): p. 253-265.

[33] Gupta, S., et al., Role of cloud ERP and big data on firm performance: a dynamic capability view theory perspective. Management Decision, 2018.

[34] Leyh, C., Critical success factors for ERP system implementation projects: A literature review. Advances in Enterprise Information Systems II, 2012: p. 45-56.

[35] Navaneethakrishnan, C., A comparative study of cloud based ERP systems with traditional ERP and analysis of cloud ERP implementation. International Journal of Engineering and Computer Science, 2013. 2(9): p. 2866-2869.

[36] Goel, M.S., R. Kiran, and D. Garg, Impact of cloud computing on ERP implementations in higher education. Institutions, 2011. 5: p. 8.

[37] Chand, S., et al. Cloud ERP Implementation Using Edge Computing. in 2018 5th Asia-Pacific World Congress on Computer Science and Engineering (APWC on CSE). 2018. IEEE.

[38] Shah, S.I.H., et al., Socio-technical factors affecting ERP implementation success in Pakistan: an empirical study. Australian Journal of Basic and Applied Sciences, 2011. 5(3): p. 742-749.

[39] Gupta, S., et al., Identification of challenges and their ranking in the implementation of cloud ERP: A comparative study for SMEs and large organizations. International Journal of Quality \& Reliability Management, 2017. 34(7): p. 1056-1072.

[40] Alajbegovic, A., V. Alexopoulos, and A. Desalermos, Factors influencing cloud ERP adoption: a comparison between SMEs and large companies. 2013.
[41] Mac-Anigboro, O. and A. Usoro, Usability in Ensuring a Successful Post Implementation Adoption of Cloud Enterprise Resource Planning Systems: A Proposed Research. Computing \& Information Systems, 2015. 19(2).

[42] Kiadehi, E.F. and S. Mohammadi, Cloud ERP: Implementation of enterprise resource planning using cloud computing technology. Journal of Basic and Applied Scientific Research, 2012. 2(11): p. 11422-11427.

\section{Appendix}

\section{Appendix-A: List of CSFs affecting cloud ERP implementation}

\begin{tabular}{|l|l|}
\hline Factors & Sources \\
\hline 1. Security & {$[14],[24],[15],[20],[28],[21],[22]$} \\
\hline 2. Project management. & {$[26],[15],[23],[20],[27],[21]$} \\
\hline 3. Communication within the company & {$[24],[26],[15],[23],[20]$} \\
\hline 4. Compliance & {$[14],[24],[15],[21]$} \\
\hline 5. Network & {$[14],[24],[15],[21]$} \\
\hline 6. Organization resistance & {$[24],[15],[23],[26]$} \\
\hline 7. Project budget & {$[26],[15],[23],[20]$} \\
\hline 8. Top management support & {$[14],[27],[26],[22]$} \\
\hline 9. Training of user & {$[14],[22],[20],[27]$} \\
\hline 10. Data integrity/quality and system testing. & {$[15],[22],[20],[27]$} \\
\hline 11. Implementation strategy & {$[15],[27],[21],[22]$} \\
\hline 12. Strategic goals and objectives & {$[15],[23],[27],[26]$} \\
\hline 13. User involvement & {$[15],[26],[14],[20]$} \\
\hline 14. Business process re-engineering & {$[15],[26],[23],[22]$} \\
\hline 15. Selection of vendor/consultant & {$[14],[20],[27],[26]$} \\
\hline 16. IT infrastructure. & {$[15],[26],[20]$} \\
\hline 17. Functionality & {$[15],[26],[21]$} \\
\hline 18. Project team & {$[14],[27],[26]$} \\
\hline 19. Trust on vendor & {$[14],[27],[26]$} \\
\hline 20. Selection of ERP package & {$[26],[15]$} \\
\hline 21. Performance expectancy & {$[25],[21]$} \\
\hline 22. Clarity of potential cost & {$[27],[21]$} \\
\hline 23. Project planning and control & {$[24]$} \\
\hline 24. Organizational culture & {$[24]$} \\
\hline 25. Knowledge base of the company & {$[24]$} \\
\hline 26. Effort expectancy & {$[25]$} \\
\hline 27. Social influence & {$[25]$} \\
\hline 28. Facilitating conditions & {$[25]$} \\
\hline 29. Age & {$[25]$} \\
\hline 30. Gender & {$[25]$} \\
\hline 31. Highest degree attained & {$[25]$} \\
\hline 32. System was customized to suite business \\
process & {$[20]$} \\
\hline 33. Readiness of organizations & {$[27]$} \\
\hline 34. Organization size & {$[21]$} \\
\hline 35. The vision of the organization & {$[21]$} \\
\hline
\end{tabular}

